Association for

Computing Machinery

July $13-14,2017$

Santa Barbara, CA, USA

Advancing Computing as a Science \& Profession

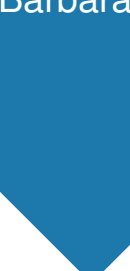

\title{
SPIN'17
}

Proceedings of the 24th ACM SIGSOFT International SPIN Symposium on

\section{Model Checking of Software}

Edited by:

Hakan Erdogmus and Klaus Havelund

Sponsored by:

ACM SIGSOFT

Supported by:

Stinger Ghaffarian Technologies, Microsoft, AppFolio, Google, NSF, UC Santa Barbara 


\section{The Association for Computing Machinery, Inc. 2 Penn Plaza, Suite 701 New York, NY 10121-0701}

Copyright (C) 2017 by the Association for Computing Machinery, Inc (ACM). Permission to make digital or hard copies of portions of this work for personal or classroom use is granted without fee provided that the copies are not made or distributed for profit or commercial advantage and that copies bear this notice and the full citation on the first page. Copyrights for components of this work owned by others than ACM must be honored. Abstracting with credit is permitted.

To copy otherwise, to republish, to post on servers or to redistribute to lists, requires prior specific permission and/or a fee. Request permission to republish from: Publications Dept. ACM, Inc.

Fax +1-212-869-0481 or E-mail permissions@acm.org.

For other copying of articles that carry a code at the bottom of the first or last page, copying is permitted provided that the per-copy fee indicated in the code is paid through the Copyright Clearance Center, 222 Rosewood Drive, Danvers, MA 01923.

\section{Notice to Past Authors of ACM-Published Articles}

ACM intends to create a complete electronic archive of all articles and/or other material previously published by ACM. If you have written a work that was previously published by ACM in any journal or conference proceedings prior to 1978, or any SIG Newsletter at any time, and you do NOT want this work to appear in the ACM Digital Library, please inform permissions@acm.org, stating the title of the work, the author(s), and where and when published.

ACM ISBN: 978-1-4503-5077-8

Additional copies may be ordered prepaid from:

$\begin{array}{ll}\text { ACM Order Department } & \text { (U.S.A. and Canada) } \\ \text { P.O. BOX 11405 } & +1-212-626-0500 \\ \text { Church Street Station } & \text { (All other countries) } \\ \text { New York, NY 10286-1405 } & \text { Fax: +1-212-944-1318 } \\ & \text { E-mail: acmhelp@acm.org }\end{array}$

Cover photo by Tony Mastres - UC Santa Barbara, looking at campus point from the air - (CUC Santa Barbara

Production: Conference Publishing Consulting

D-94034 Passau, Germany, info@conference-publishing.com 


\section{Message from the Chairs}

This volume contains the proceedings of the 24th International Spin Symposium on Model Checking of Software (Spin 2017), which took place at the University of California, Santa Barbara, on July 13 and 14, 2017. Spin 2017 was collocated with the International Symposium on Software Testing and Analysis (ISSTA), and was organizationally sponsored by the Association of Computing Machinery (ACM) and the Special Interest Group on Software Engineering (SIGSOFT). Adding to the great synergy between ISSTA and Spin was another sister workshop: the 7th International Challenge on the Rigorous Examination of Reactive Systems (RERS). With the additional contribution of Workshop on Testing Embedded and Cyber-Physical Systems (TECPS), the four events resulted in a week-long program packed with activities that cross-cut a wide range of interrelated interests.

The Spin symposia bring together researchers and practitioners working in automated, tool based techniques for the analysis, verification, and validation of software systems, including models and programs. The symposia debuted as a workshop series in 1995 in Montréal, Canada. Its initial focus was on the Spin model-checker, developed by Gerard Holzmann, and related approaches. Over time, the focus expanded from model checking - the process of checking whether a given structure, often representing a concurrent system, satisfies a correctness property - to include other rigorous and automatable analysis approaches. Furthermore, the focus expanded from analysis of abstract models to also include actual software artifacts (programs and code).

For the 2017 edition of Spin, we solicited contributions addressing a broad scope of topics, including: formal verification techniques for automated analysis of software; formal analysis for modeling languages such as UML and statecharts; formal specification languages; automated theorem proving, including SATand SMT-based techniques; compiler verification; abstraction and symbolic execution techniques; static analysis and abstract interpretation; combining verification techniques; modular and compositional verification; verification of timed and probabilistic systems; automated testing using advanced analysis techniques; combination of static and dynamic analyses; derivation of code, specifications, and test cases via formal analyses; engineering and implementation of software verification and analysis tools; and case studies, benchmarks, and comparative studies addressing the symposium topics.

Spin 2017 considered two types of contributions: full papers describing mature work and complete results, and short papers addressing tools and technologies, experiences with lessons learned, new and emerging ideas, and work in progress with preliminary results. We received 30 full papers and 16 short papers in response to our call. These submissions represented 26 countries of origin by author or co-author affiliation, reflecting the international nature of Spin. They were, in order of number of submissions: USA (13), Germany (6), France (6), UK (4), Netherlands (4), China (3), Austria (2), Japan (2), Iran (2), Czech Republic (2), Australia (1), Bangladesh (1), Brazil (1), Colombia (1), Denmark (1), Finland (1), Greece (1), Italy (1), India (1), New Zealand (1), Romania (1), Singapore (1), South Africa (1), Spain (1), Sweden (1), and Turkey (1). Of the 46 submissions received, six had an industrial author or co-author and nine had an author or co-author affiliated with a government organization or research laboratory. The remaining 31 had academic authors. 
From this set, an international program committee of 34 members selected 16 full papers and five short papers for inclusion in the program, amounting to a $45.7 \%$ overall acceptance rate. Each submission was reviewed by at least three program committee members. The accepted papers represent a total of 19 countries of origin by author or co-author affiliation. In order of number of accepted papers, they were: USA (5), France (5), UK (3), Netherlands (3), Germany (2), Austria (1), China (1), Colombia (1), Denmark (1), Finland (1), Greece (1), Italy (1), Iran (1), New Zealand (1), Romania (1), Singapore (1), South Africa (1), Spain (1), and Sweden (1).

Led by the awards chair Corina Pasareanu, the program committee identified a best paper in each submission category. The Best Full Paper Award went to Wytse Oortwijn, Tom van Dijk, and Jaco van de Pol for their paper titled "Distributed Binary Decision Diagrams for Symbolic Reachability." The Best Short Paper Award went to Lucas Wagner, David Greve, and Andrew Gacek for "SIMPAL: A Compositional Reasoning Framework for Imperative Programs." Congratulations to the winners!

Spin 2017 featured keynote addresses by three distinguished speakers on highly applied topics. Domagoj Babic (Google Inc., USA) gave a talk titled "SunDew: Systematic Automated Security Testing," describing an automated test generation framework developed at Google and focused on finding security bugs in $\mathrm{C} / \mathrm{C}++$ code. Byron Cook (Amazon Web Services, USA, and University College London, UK) gave a talk titled "Automated Formal Reasoning about Amazon Web Services," describing current efforts at Amazon Web Services that use automated formal verification tools, specifically for replaying proofs when software artifacts are modified. Gerard Holzmann (Nimble Research, USA) gave a talk titled "Cobra: Fast Structural Code Checking" (full paper included in proceedings), describing a tool for efficient, interactive static analysis of large code bases. We are indebted to our excellent keynote speakers for taking the time from their busy lives to share their insights. In addition, the proceedings contain a contribution from the RERS organizers titled "The RERS 2017 Challenge and Workshop," on the verification challenge that focused on Linear Temporal Logic and reachability properties of reactive systems.

Spin 2017 honored steering committee member Gerard Holzmann. Dr. Holzmann's contributions deserve special recognition. His model checker Spin has had a major influence on the community, winning him the 2001 ACM Software Systems Award. He has also been a constant driving force behind the Spin workshops, and later the symposia, since the inception of the first event 21 years ago. We express our deepest gratitude to Dr. Holzmann for his service and pioneering work.

We thank all authors for submitting their work to Spin 2017, resulting in one of the highest number of submissions in Spin's history. The program committee and external reviewers worked diligently in evaluating the submissions. We appreciate greatly their hard work and high-quality reviews. The Spin steering committee provided valuable guidance, especially during the review process, helping us tackle some contentious issues. All of them, in addition, participated in the program committee. Our sincerest thanks also go to the other members of the organizing committee. Corina Pasareanu, of Carnegie Mellon University and NASA Ames, served as awards chair and managed the process for best paper selections. Yliés Falcone, of Université Grenoble Alpes and Inria, served as publicity chair, helping us reach out to the community and attract a high number of submissions.

We worked closely with the ISSTA 2017 organization, pooling our resources for a successful joint event. 
The National Science Foundation and Google provided travel and financial support for students, some of whom would not have been able to attend without their help. Our gold sponsor Stinger Gaffarian Technologies and our silver sponsors Microsoft and Appfolio provided cash contributions, which enabled us to reduce registration fees and keep costs at bay. We are grateful to ACM and SIGSOFT for being our organizational sponsors and giving us their full support. The University of California, Santa Barbara, and its College of Engineering and Computer Science Department supplied the wonderful venue in the beautiful surroundings of Southern California. Finally, we thank Tevfik Bultan, ISSTA general chair, and Nicolás Rosner, ISSTA Publicity Chair, for their assistance in making Spin 2017 a success.

Hakan Erdogmus and Klaus Havelund

Spin 2017 Program Co-Chairs

Support for the preparation of these proceedings was in part provided by the Jet Propulsion Laboratory, California Institute of Technology, under a contract with the National Aeronautics and Space Administration. 


\section{SPIN 2017 Organization}

\section{Organizing Committee}

\section{Program Co-Chairs}

Hakan Erdogmus

Klaus Havelund

Awards Chair

Corina Pasareanu

Publicity Chair

Yliès Falcone

\section{Steering Committee}

Dragan Bosnacki

Susanne Graf

Gerard Holzmann

Stefan Leue

Willem Visser

\section{Program Committee}

Erika Abraham

Christel Baier

Tom Ball

Ezio Bartocci

Dirk Beyer

Armin Biere

Dragan Bosnacki

Zmago Brezocnik

Sagar Chaki

Alessandro Cimatti

Lucas Cordeiro

Patrice Godefroid

Susanne Graf

Radu Grosu

Arie Gurfinkel

Gerard Holzmann

Rajeev Joshi

Sarfraz Khurshid

Kim Larsen
Carnegie Mellon University, USA

Jet Propulsion Laboratory, California Institute of Technology, USA

NASA Ames Research Center and Carnegie Mellon University, USA

Université Grenoble Alpes and Inria, France

Eindhoven University of Technology, Netherlands

Verimag, France

Nimble Research, USA

University of Konstanz, Germany

Stellenbosch University, South Africa

RWTH Aachen University, Germany

TU Dresden, Germany

Microsoft Research, USA

Technische Universität Wien, Austria

LMU Munich, Germany

Johannes Kepler University, Austria

Eindhoven University of Technology, Netherlands

University of Maribor, Slovenia

Carnegie Mellon Software Engineering Institute, USA

Fondazione Bruno Kessler, Italy

University of Oxford, UK

Microsoft Research, USA

Verimag, France

Vienna University of Technology, Austria

University of Waterloo, Canada

Nimble Research, USA

Jet Propulsion Laboratory, California Institute of Technology, USA

University of Texas at Austin, USA

Aalborg University, Denmark 
Stefan Leue

Alice Miller

Corina Pasareanu

Doron Peled

Neha Rungta

Theo Ruys

Scott Smolka

Scott Stoller

Jun Sun

Oksana Tkachuk

Stavros Tripakis

Willem Visser

Farn Wang

Michael Whalen

Anton Wijs

\section{Additional Reviewers}

Deepayan Bhattacharjee

Richard Defrancisco

Stefan Jaksic

Rody Kersten

Florian Lorber

Eric Mercer

Dung Phan

Sander de Putter

Konstantin Selyunin

Michael Tautschnig
University of Konstanz, Germany

University of Glasgow, UK

NASA Ames Research Center and Carnegie Mellon University, USA

Bar Ilan University, Israel

Amazon Web Services, USA

RUwise, Netherlands

Stony Brook University, USA

Stony Brook University, USA

Singapore University of Technology and Design, Singapore

NASA Ames Research Center, USA

Aalto University, Finland and University of California Berkeley, USA

Stellenbosch University, South Africa

National Taiwan University, Taiwan

University of Minnesota, USA

Eindhoven University of Technology, Netherlands
Taejoon Byun

Nima Dini

Peter Gjøl Jensen

Martin Kölbl

Anna Lukina

Thomas Neele

Quoc-Sang Phan

Giles Reger

Jiri Srba

Jana Tumova
Gerogiana Caltais

Elaheh Ghassabani

Panagiotis Katsaros

Chenguang Liu

Ravi Mangal

Nicola Paoletti

Mathias Preiner

Alena Rodionova

Danielle Stewart

Tim Willemse 


\section{Sponsors}

\section{\begin{tabular}{|l}
$\square$ \\
$\square$
\end{tabular} \\ STINGER GHAFFARIAN TECHNOLOGIES}
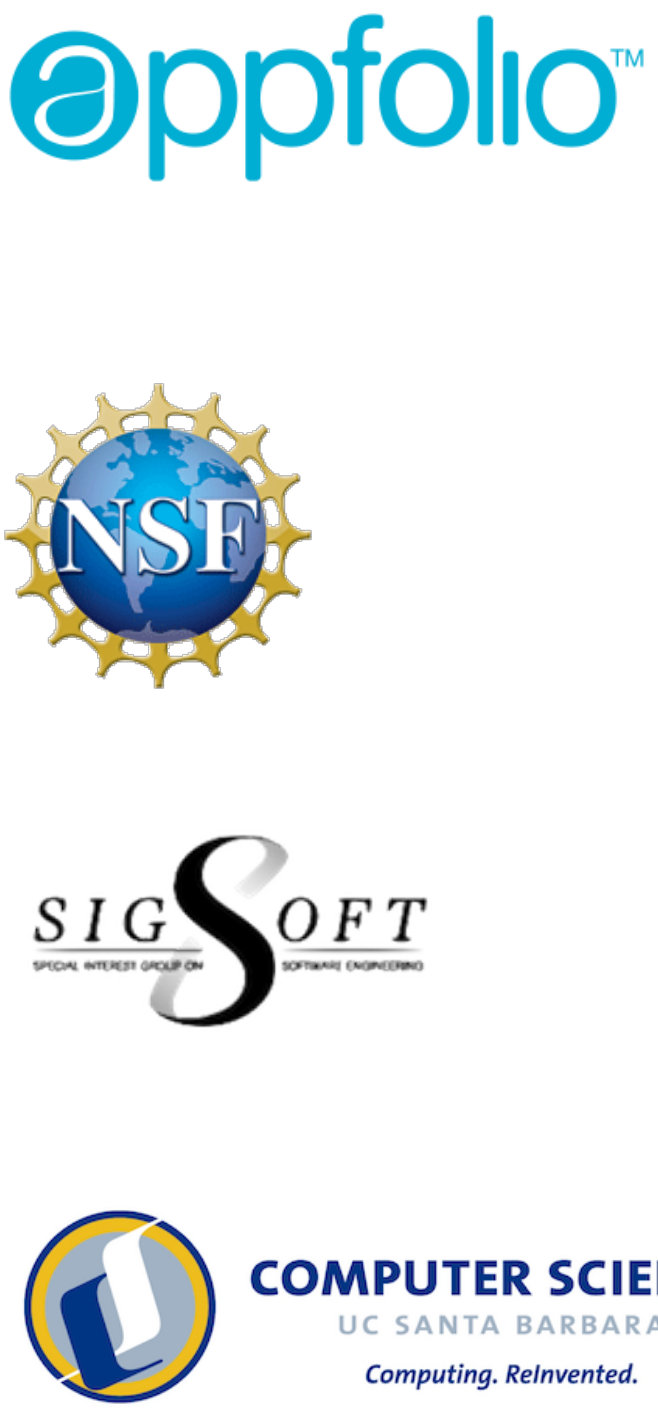

COMPUTER SCIENCE

UC SANTA BARBARA

Computing. Relnvented.
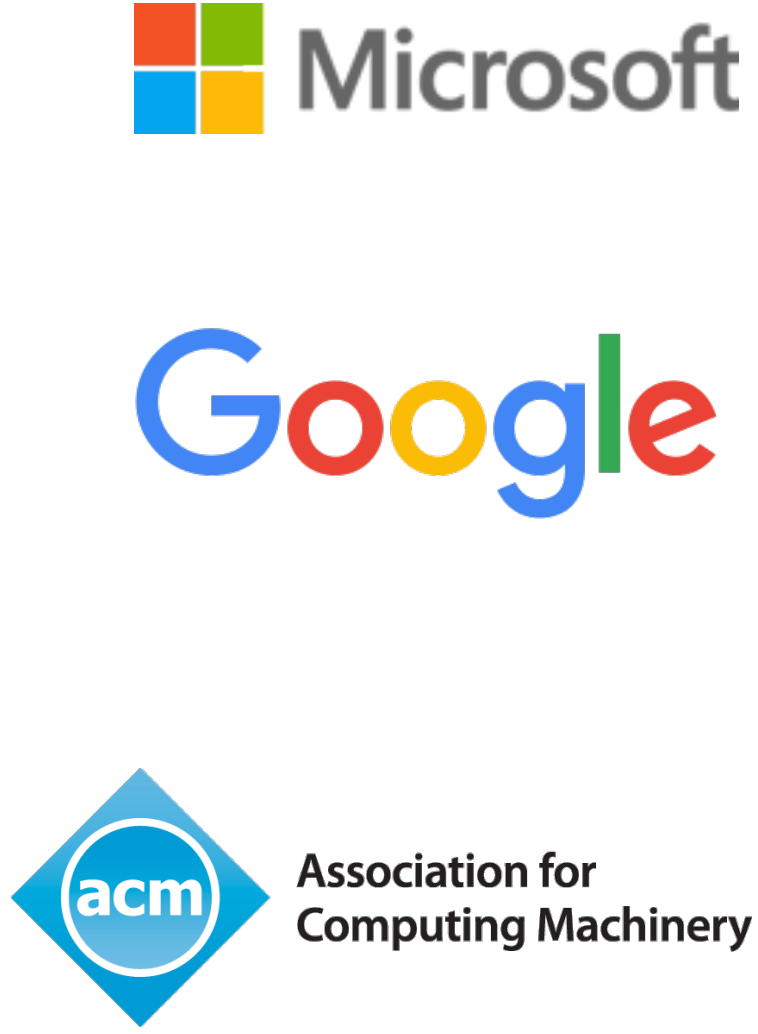

(3) UC SANTA BARBARA 


\section{Contents}

\section{Frontmatter}

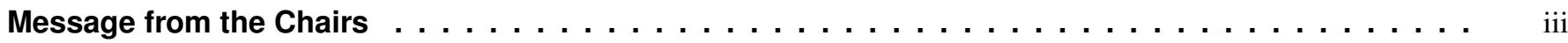

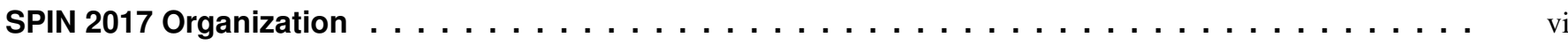

Sponsors $\ldots \ldots \ldots \ldots \ldots \ldots \ldots \ldots \ldots \ldots \ldots \ldots \ldots \ldots \ldots \ldots \ldots \ldots \ldots$ viii

\section{Invited Papers}

Cobra: Fast Structural Code Checking (Keynote)

Gerard J. Holzmann — Nimble Research, USA . . . . . . . . . . . . . . . . . . . . . . . . 1

Automated Formal Reasoning about Amazon Web Services (Keynote)

Byron Cook — Amazon Web Services, USA; University College London, UK . . . . . . . . . . . . . . . . . . 9

SunDew: Systematic Automated Security Testing (Keynote)

Domagoj Babic — Google, USA . . . . . . . . . . . . . . . . . . . . . . . .

\section{Reports}

\section{The RERS 2017 Challenge and Workshop (Invited Paper)}

Marc Jasper, Maximilian Fecke, Bernhard Steffen, Markus Schordan, Jeroen Meijer, Jaco van de Pol, Falk Howar, and Stephen F. Siegel — Lawrence Livermore National Laboratory, USA; TU Dortmund, Germany; University of Twente, Netherlands; TU Clausthal, Germany; University of Delaware, USA ． . . . . . . . . . . . . . . . . . .

\section{Symbolic Verification}

Distributed Binary Decision Diagrams for Symbolic Reachability

Wytse Oortwijn, Tom van Dijk, and Jaco van de Pol - University of Twente, Netherlands; JKU Linz, Austria . . . .

\section{Model Checking I}

Addressing Challenges in Obtaining High Coverage When Model Checking Android Applications Heila Botha, Oksana Tkachuk, Brink van der Merwe, and Willem Visser — Stellenbosch University, South Africa; NASA Ames Research Center, USA . . . . . . . . . . . . . . . . . . . . . . . . . . . . . . .

LeeTL: LTL with Quantifications over Model Objects

Pouria Mellati, Ehsan Khamespanah, and Ramtin Khosravi - University of Tehran, Iran; Reykjavik University, Iceland

Explicit State Model Checking with Generalized Büchi and Rabin Automata

Vincent Bloemen, Alexandre Duret-Lutz, and Jaco van de Pol - University of Twente, Netherlands; LRDE, France .

Code Verification

Increasing Usability of Spin-Based C Code Verification using a Harness Definition Language: Leveraging Model-Driven Code Checking to Practitioners

Daniel Ratiu and Andreas Ulrich — Siemens, Germany . . . . . . . . . . . . . . . . . . . . . . .

\section{Runtime Enforcement}

\section{Runtime Enforcement using Büchi Games}

Matthieu Renard, Antoine Rollet, and Yliès Falcone — LaBRI, France; Bordeaux INP, France; University of Bordeaux, France; Grenoble Alpes University, France; Inria, France; CNRS, France; Laboratoire d'Informatique de Grenoble, France . . . . . . . . . . . . . . . . . . . . . . . . . . . . .

Runtime Enforcement of Reactive Systems using Synchronous Enforcers

Srinivas Pinisetty, Partha S. Roop, Steven Smyth, Stavros Tripakis, and Reinhard von Hanxleden - Aalto University, Finland; University of Gothenburg, Sweden; University of Auckland, New Zealand; University of Kiel, Germany; University of California at Berkeley, USA . . . . . . . . . . . . . . . . . . 


\section{Model Checking - Short Papers}

SIMPAL: A Compositional Reasoning Framework for Imperative Programs

Lucas Wagner, David Greve, and Andrew Gacek — Iowa State University, USA; Rockwell Collins, USA . . . . . .

Verification-Driven Development of ICAROUS Based on Automatic Reachability Analysis: A Preliminary Case Study

Marco A. Feliú, Camilo Rocha, and Swee Balachandran — National Institute of Aerospace, USA; Pontificia Universidad Javeriana, Colombia . . . . . . . . . . . . . . . . . . . . . . . . . . . . .

Formal Verification of Data-Intensive Applications through Model Checking Modulo Theories

Marcello M. Bersani, Francesco Marconi, Matteo Rossi, Madalina Erascu, and Silvio Ghilardi — Politecnico di Milano, Italy; Institute e-Austria Timisoara, Romania; West University of Timisoara, Romania; University of Milan, Italy . .

\section{Program Synthesis}

Practical Controller Synthesis for MTL<sub>0,\&\#8734;</sub>

Guangyuan Li, Peter Gjøl Jensen, Kim Guldstrand Larsen, Axel Legay, and Danny Bøgsted Poulsen — Institute of Software at Chinese Academy of Sciences, China; University at Chinese Academy of Sciences, China; Aalborg University, Denmark; Inria, France; University of Kiel, Germany . . . . . . . . . . . . . . . . . . . . . . . . .

An Ordered Approach to Solving Parity Games in Quasi Polynomial Time and Quasi Linear Space John Fearnley, Sanjay Jain, Sven Schewe, Frank Stephan, and Dominik Wojtczak - University of Liverpool, UK; National University of Singapore, Singapore . . . . . . . . . . . . . . . . . . . . . . . . . .

A Hot Method for Synthesising Cool Controllers Idress Husien, Nicolas Berthier, and Sven Schewe - University of Liverpool, UK . . . . . . . . . . . .

\section{Model Checking II}

Backward Coverability with Pruning for Lossy Channel Systems

Thomas Geffroy, Jérôme Leroux, and Grégoire Sutre - University of Bordeaux, France; CNRS, France; LaBRI, France Model Learning and Model Checking of SSH Implementations

Paul Fiterău-Broştean, Toon Lenaerts, Erik Poll, Joeri de Ruiter, Frits Vaandrager, and Patrick Verleg — Radboud University Nijmegen, Netherlands . . . . . . . . . . . . . . . . . . . .

CARET Model Checking for Malware Detection Huu-Vu Nguyen and Tayssir Touili - University of Paris Diderot, France; LIPN, France; CNRS, France; University of Paris North, France . . . . . . . . . . . . . . . . . . . . . . . .

\section{Program Sketching}

\section{EdSketch: Execution-Driven Sketching for Java}

Jinru Hua and Sarfraz Khurshid — University of Texas at Austin, USA . . . . . . . . . . . . . .

\section{Testing}

Stateless Model Checking of the Linux Kernel's Hierarchical Read-Copy-Update (Tree RCU)

Michalis Kokologiannakis and Konstantinos Sagonas - National Technical University of Athens, Greece; Uppsala

University, Sweden . . . . . . . . . . . . . . . . . . . . . . .

Optimizing Parallel Korat Using Invalid Ranges

Nima Dini, Cagdas Yelen, and Sarfraz Khurshid — University of Texas at Austin, USA . . . . . . . . . . .

\section{Testing - Short Papers}

Guided Test Case Generation for Mobile Apps in the TRIANGLE Project: Work in Progress

Laura Panizo, Alberto Salmerón, María-del-Mar Gallardo, and Pedro Merino - University of Málaga, Spain . . . .

ExpoSE: Practical Symbolic Execution of Standalone JavaScript

Blake Loring, Duncan Mitchell, and Johannes Kinder — Royal Holloway University of London, UK . . . . . . . .

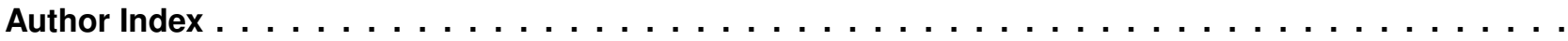

